\title{
The value of information with heterogeneous agents and partially revealing prices
}

\author{
Juan Carlos Hatchondo \\ University of Rochester
}

May 25, 2004

\begin{abstract}
This paper studies how the arrival of more precise information affects welfare in an economy with incomplete and differential information. We consider a single period, pure exchange economy with aggregate uncertainty in which agents show different attitudes towards risk: wealthy individuals are 'de facto' less risk averse than poor individuals. The first ones can then partially insure the last ones, allowing for mutual gains from trade. Agents ignore the actual probability distribution over the states. Instead, they learn from the market price and private signals, which in fact accounts for the presence of heterogeneous beliefs. In equilibrium, the dispersion in beliefs introduces an adverse effect: risk-taking agents are more pessimistic than the rest. This limits the possibilities to share risks and has a negative impact on welfare. The arrival of more precise information has therefore a double effect: it weakens the adverse effect on trade (as risk-taking agents become more optimistic, they offer more insurance) at the same time that it strengthens the Hirshleifer effect (agents are no longer able to insure against news that have already arrived). The first effect fosters and the second one discourages risk-sharing trades. The paper discusses in detail a case where the positive effect on trade offsets the negative effect. This lets us conclude that in an economy with partial information, agents' welfare may increase upon the receipt of more precise information. Even though the result looks intuitive, most of the previous literature has focused on the case with homogeneous beliefs. In such a framework, only the Hirshleifer effect is at work, and thus better information typically leads to a decrease in welfare.
\end{abstract}

Keywords: Information and Welfare, Heterogeneous Beliefs, Partially Revealing Prices

JEL Classification: $D 8$ 


\section{Introduction}

Thanks to the explosive development of the Internet and the World Wide Web, the last decade has witnessed a dramatic change in the technology to access information. At the same time, the advances in the computer technology has made possible to process a significantly larger amount of information than it was possible a few years ago. The changes are particularly shocking when we look at financial markets. Nowadays, any individual can get access to specialized reports, series of data or the latest news before trading. Thus, no one would question that market participants have become much more sophisticated compared to what they were twenty years ago. The question remains whether these changes have been accompanied by an increase in welfare. The intuition suggests that more precise information should lead to better decisions and hence, should be welfare improving. The examples below, however, challenge this common sense view and illustrate cases where the receipt of more information may be harmful.

Lerman et al. (1996) describe the results of a survey conducted among members of families with BRCA1-linked hereditary breast-ovarian cancer. ${ }^{1}$ They find that $57 \%$ of the individuals interviewed declined to take a free BRCA1 test. One of the reasons listed against being tested was the possibility of losing health insurance. Quaid and Morris (1993) reports a similar behavior in a sample of individuals who were offered a free test of Huntington's disease.

Since 1994 the Federal Reserve announces interest rate changes at pre-scheduled and publicly available dates. The decisions are made public after the meetings of the Federal Open Market Committee. The Fed deviated from this scheme in January and April of 2001, when it decided to cut interest rates well before the next scheduled meeting. Banerjee and Seccia (2002) find evidence of abnormal volumes of trade in interest rate futures the day before the scheduled meeting, but they find no evidence of excess trade before the two unscheduled announcements. They conclude that the two unexpected interest rate cuts might have had a negative impact on welfare, if a fraction of the abnormal trade is motivated by hedging purposes. In those two opportunities, agents did not have the possibility to insure against interest rate changes. This example illustrates a theoretical result in Sulganik and Zilcha (1996). They argue that when future markets are available, in which case agents can share risks, the value of information may not always be positive. A similar conclusion is obtained by Drees and Eckwert (2003). They argue

\footnotetext{
${ }^{1}$ The isolation of the BRCA1 gene allows to learn if the individual carries a cancerpredisposing mutation that increases the probability of developing breast or ovarian cancer.
} 
that more transparency in the foreign exchange market may reduce welfare when agents can hedge the currency risk.

The previous papers illustrate a general principle: the arrival of more accurate information may be harmful if it precludes risk sharing trades, agents cannot insure against events that are not longer uncertain. Drèze (1960) was the first to identify the possibility that information may have a negative value, but the result is commonly known in the literature as the 'Hirshleifer effect'. Hirshleifer (1971) formalized Drèze's argument using a general equilibrium framework. Consider an Edgeworth box economy with one good, two agents and two states of the world. The state probabilities are common knowledge. In such a framework, if markets open before the state realization is known, agents trade to a point on the contract curve. But if markets open after the state of the world has been publicly disclosed, no trade takes place. In that case, better information leads to a worse consumption allocation.

The present paper analyzes how the receipt of more precise information affects welfare in a pure exchange economy with incomplete information and one round of trading. The model has two main features: there is uncertainty about the state that will be realized in the future; and agents are affected asymmetrically in different states. The latter allows them to share risks and partially insure against the states where they are unlucky. The structure of the model captures an important feature of financial markets: agents trade to share risks, but also because they have different expectations about the assets being traded.

We consider a pure exchange economy where agents can invest in two assets: a risk free bond and a risky asset, namely a tree. The tree pays either high or low dividends. The ex-ante probabilities of these events are not known, but each agent receives private signals about the tree. Each signal can be either good or bad. The probability of receiving a good signal depends on the probability that the tree pays high dividends. The last feature is what makes the signals informative. Agents also receive a riskless endowment that may take either a high or low value. The fraction of agents receiving a high riskless endowment, the rich ones, is also unknown. We assume agents share the same preferences, which can be represented by a concave utility function with decreasing coefficient of absolute risk aversion. Thus, rich individuals are 'de-facto' less risk averse than poor individuals, so the former tend to insure the latter. Markets open before the tree pays off. The equilibrium price of the risky asset depends on two variables: the probability of high dividends (which determines the distribution of signals across agents) and the 
fraction of rich agents. Agents face a nontrivial signal extraction problem: they see a price but cannot infer its two underlying determinants. Their posterior belief is based on three pieces of information: the price, the private signals about the tree and the individual realization of the riskless endowment. If agents could observe the fraction of rich agents before trading, they would be able to use the equilibrium price to infer the actual probability with which the tree pays high dividends. In our framework, however, agents are not able to infer that probability because the distribution of endowments is also unknown.

In the extreme case where everyone is fully informed, the model predicts that rich agents insure poor agents by purchasing risky assets and selling risk free bonds. This prediction may not hold in the partial information economy considered in the present paper. The reason is the following one. If an agent receives a low endowment, he infers that the fraction of poor agents in the economy is relatively high. In other words, agents who are hit with a shock that induces them to sell stocks (i.e. a low endowment), believe that there are many others in the same situation. So a low endowment serves as a signal that the excess demand of stocks is low. Conversely, rich agents believe that the excess demand of stocks is high. This leads to different interpretations of the market price. For a given price, poor (rich) agents perceive there are more (less) agents who dislike risks, so they infer that the reason why agents demand stocks is because they offer a 'high' (low) expected return. In summary, agents who are hit with a low endowment tend to be more 'optimistic' than agents with high endowments. This effect dampens the incentives to share risks. As agents become more sophisticated and acquire more precise information, i.e. more signals, the dispersion of the beliefs shrinks, which tends to offset the previous negative effect.

We find that more precise information about the underlying source of risk can enhance or reduce the possibilities to share risks among agents, leading to a increase or decrease in welfare, respectively. The Hirshleifer effect is still present in our model, but there is another channel through which information affects the equilibrium allocation: the dispersion of beliefs. The model describes a case where beliefs' heterogeneity is such that discourages trading. In those cases, better information reduces the dispersion of beliefs and hence, has a positive welfare effect.

If the asymmetric information assumption were abandoned and agents received common signals, instead of private signals, the model would belong to the set of economies studied in Schlee (2001). We would expect therefore to observe a decrease in individual welfare after the arrival of better information. 
The present paper, however, offers a different conclusion, showing that Schlee's result depends crucially on the homogeneity of beliefs and do not extend to economies with differential information.

\section{$1.1 \quad$ Related Literature}

Several authors have studied more general frameworks to test the validity of Hirshliefer's result. Marshall (1974) analyzes the value of public information when individuals can trade before and after the arrival of information. He concludes that:

If the impact of information is insured before its arrival, that insurance precludes further trade based on the news. ... In all, the information has no impact on distribution, no impact on satisfaction, and hence, no value.

In the contrary case when the news must arrive before its impact is insured in a preliminary market, the information is harmful. People can afford to pay something to suppress the news or to delay its arrival. ... Public information in this situation is harmful; at best its impact can be counteracted by prior insurance. ${ }^{2}$

A similar conclusion is obtained in Ng (1975) and Green (1981). Hakansson et al. (1982) provide sufficient and necessary conditions for public information to have positive social value. ${ }^{3}$ Their results, though, rely on the fact that agents can trade prior to the arrival of information. If that market is missing, they cannot rule out the possibility that better information may decrease social welfare. Schlee (2001) focuses on the last case, where individuals trade after the receipt of information. He concludes that better information typically reduces every agent's welfare in any of the following situations: there is no aggregate risk; some agents are risk neutral; or the economy behaves as if there were a representative agent.

It must be said that with the exception of Green (1981), the papers described before assume there is a common signal in the economy. Agents update their beliefs after the signal is announced and then trade. The common signal assumption simplifies the analysis considerably as agents do not need to learn from the prices. In effect, the latter do not convey any information that is not contained in the signal. This simplifies the analysis considerably but, as the present paper shows, it may bias the conclusions. Besides, the assumption that individuals hold homogeneous beliefs lacks empirical support.

\footnotetext{
${ }^{2}$ Marshall (1974), p 380.

${ }^{3}$ They show that if the initial endowments are an equilibrium allocation without information, then the arrival of public information leads to a Pareto superior consumption allocation in any of the following cases: the marginal rates of substitutions are not equalized across agents, or there is sufficient asymmetry in the posterior beliefs.
} 
The assumption that prices reveal valuable information has been extensively studied in the literature. The first generation of models developed by Grossman (1976), Grossman and Stiglitz (1980), Hellwig (1980), Diamond and Verrecchia (1981), Verrecchia (1982), and Admati (1985) prevents prices from fully revealing the fundamentals of the economy by assuming the existence of liquidity traders. The latter display a random behavior and distort the information conveyed by the price. Even though these models have allowed to obtain valuable insights in many areas, the assumption of liquidity traders makes them unsuitable for welfare analysis. Among the papers that have been able to obtain partially revealing prices without resorting to noise traders, we should mention Ausubel (1990a). Since the structure of our model share some similarities with his work, we defer a more detailed discussion until the end of section 2 .

There is another family of models, considered in Bhattacharya and Matthew (1991), Rahi (1996) and Marín and Rahi (2000), that also allow for the existence of partially revealing equilibria without assuming the presence of noise traders. These models introduce an asymmetry in the information held by agents, i.e., they assume there is a group that is more informed than the rest. This generates and adverse selection effect: uninformed agents reduce their participation in the market because of their informational disadvantage. In that framework, the receipt of more precise information not only strengthens the Hirshleifer effect but also dampens the adverse selection effect. Thus, more information does not necessarily lead to a decrease in welfare. Even though the conclusion is similar to the one described in the present paper, the mechanisms explaining the result are different. The disparities in the level of information appears as a sensible assumption when we consider trades on assets issued by private companies, in which case there may be leakages of privileged information. However, it is a harder assumption to justify when we look at trades on assets linked to aggregate variables, like interest rates or exchange rates futures. Besides, the modelling strategy followed in the present paper allows for a straightforward comparison with the existing literature. In fact, our model could be mapped into the set of economies considered by Schlee (2001) if agents received public signals instead of private signals.

Among the papers that have explicitly analyzed the relationship between welfare and information in economies with partially revealing equilibria, we find Berk (1997). He analyzes a simple dynamic game and concludes that it is possible for an equilibrium to exist in which agents choose to purchase information even if all agents, including the agents who purchased the information, are made strictly 
worse off from an ex ante perspective.

Citanna and Villanacci (2000) study a class of models with partially revealing prices, multiple goods, asymmetric information and heterogeneous wealth. They find that welfare may increase after the arrival of more precise information. The reason is that wealth effects due to price changes may outweigh the Hirshleifer effect. Gottardi and Rahi (2001) also analyze a model with asymmetric information and reach a similar conclusion. Besides, they are able to disentangle the different channels through which the arrival of better information affects welfare. ${ }^{4}$

The present paper also utilizes a model with partially revealing prices and asymmetric information to assess the value of public information. Unlike Citanna and Villanacci (2000), the beliefs' updating scheme is endogenous and is interlinked across agents. Also, their results cannot be extended to the present paper, since they depend on a countable number of states and more than two goods.

Finally, we should mention that a positive relationship between the precision of public information and welfare can also be observed once the endowment economy framework is abandoned. In a model with production, the early arrival of public information has an additional effect: it may allow for better investment decisions. In order to relativize the negative impact of information, Hirshleifer (1971) provides an example where the last effect dominates and better information is welfare enhancing. In a recent paper, Eckwert and Zilcha (2003) study more formally the value of information in an economy with production. They assume that the production function is subject to a productivity shock that consists of the sum of two random variables. Agents have partial information about the realization of one of these variables and trade on that information. The authors show that information about non-tradable risks has always a positive value.

The paper is organized as follows. Section 2 sets out the model and defines the equilibrium concept used in the paper. Section 3 describes the criteria for choosing the baseline parametrization. Section 4 presents the results. Finally, we conclude in Section 5.

\footnotetext{
${ }^{4}$ The structure of the model considered in their paper allows only for a fully revealing equilibrium. Thus, in order for the asymmetric information assumption to play any role, they have to use a definition of equilibrium that is less restrictive than the standard rational expectations equilibrium concept used in the literature.
} 


\section{The model}

The paper applies the baseline model presented in Hatchondo et al. (2003) to study how the receipt of more accurate information affects agents' welfare. Even though the model has a simple structure, it does not allow for a tractable analytical solution, so we must rely thus on numerical techniques to characterize the equilibrium.

We consider a pure exchange economy with asymmetric information and heterogeneous agents. There is a single risky asset in the economy: a tree. The tree pays high dividends with probability $\nu$ and low dividends with probability $1-\nu$. The tree pays only once and then dies. There is a measure 1 of agents in the economy and everybody is initially entitled with a share of the tree. Agents also receive a riskless endowment, though some of them are luckier than others, i.e., a fraction $\phi$ of the population receives a high endowment, while a fraction $1-\phi$ receives a low endowment.

The parameters $\nu$ and $\phi$ are drawn from a joint probability distribution $F(\nu, \phi)$. The latter is common knowledge. The random variable $\nu$ takes values on the unit interval $I \equiv[0,1]$. The random variable $\phi$ is discrete and takes values on $\Phi=\left\{\phi_{l}, \phi_{h}\right\}$.

Agents are not able to observe the realizations of those variables but receive informative signals about the tree. Each signal can be either good or bad. Every agent receives $n$ number of signals. The realizations of the signals are drawn from a Binomial distribution with parameter $\nu$. As the number of signals increases, the information agents receive becomes more accurate. The case where $\nu$ is common knowledge corresponds to an infinite number of signals.

The assumption described in the previous paragraph deserves two comments. First, we acknowledge that there exist other mechanisms that can be used to model the transmission of information instead of the binary signals structure. We choose the latter for the sake of simplicity. Second, the paper is silent about how the number of signals is determined. In order to circumvent this limitation, the model could be interpreted as a case where agents decide the number of signals they purchase. ${ }^{5}$ The process of acquisition of information is not the main focus of this paper, so it is not modelled explicitly.

All the action takes place in a single period. Markets open in the morning, the tree pays off in the afternoon and agents consume at the end of the day. In the absence of trade, agents consume their

\footnotetext{
${ }^{5}$ See Verrecchia (1982) for a case where agents decide the precision of the information acquired.
} 
endowments and dividends paid by the tree. Actually, this would be the equilibrium allocation if there was no heterogeneity across agents. This is not the case in the present framework, though. Poor agents have a stronger preference for consumption smoothing than wealthy individuals, so there are gains from trade. 6

Agents can transfer resources freely across the two states of nature that can be realized, i.e., whether the tree pays high or low dividends. This means that consumers can trade in two Arrow-Debreu securities. One of them pays 1 unit of the consumption good if the high dividends state is realized. Otherwise, it pays zero. The other security only pays (1 unit) in the low dividends state. There is only one price to be determined: the relative price between these two securities.

The previous market structure would be enough to attain an efficient allocation in an economy with full information. This is no longer true in the present example. As will become clear in the next section, an extra asset (and hence another market) would be necessary to attain an efficient allocation with the information structure assumed before. The present paper studies the case where that is not possible: markets are incomplete.

The equilibrium price depends on $\nu$ and $\phi$. A higher value of $\nu$ means that the high dividend state is more likely, which makes the contingent claim paying in that state more valuable. A higher $\phi$ implies that a lower fraction of agents needs insurance, which reduces the demand for contingent claims paying in the low state.

The critical assumption made in the paper is that agents are fully rational and use the information pooled by the equilibrium price when they update their beliefs. Agents not only learn from their private signals, but also understand how the price is determined in equilibrium. This allows them to make inferences about the realizations of $\nu$ and $\phi$ once they have observed the market price. In addition, the endowment realization also conveys valuable information, as will be described below. Finally, the paper assumes agents do not behave strategically. They take the price and everyone else's behavior as given. This is justified on the ground that there is a 'large' number of agents, so each individual does not exert any influence on aggregate variables.

\footnotetext{
${ }^{6}$ This result is true if the utility function is concave and shows a decreasing coefficient of absolute risk aversion. The latter is defined as $\frac{-u^{\prime \prime}(c)}{u^{\prime}(c)}$. The utility function assumed in the present paper (logarithmic) satisfies both properties.
} 


\subsection{Definition of equilibrium}

Agents maximize expected utility of consumption. They do not know the actual state probabilities, so they use the information contained in the private signals, the endowment and the market price to refine their belief about $\nu$. We assume the belief consists of the expectation of $\nu$ conditional on the price and the agents' private information. Formally, let $\mathcal{I}_{i}$ denote the private information set of agent i, specifically, the signals and endowment received. His belief about $\nu$ is denoted by $\tilde{\nu}^{i}$, where

$$
\tilde{\nu}^{i}\left(\mathcal{I}, p, \mathcal{P}^{i}(\cdot)\right)=E\left[\nu \mid \mathcal{I}_{i}, p, \mathcal{P}^{i}(\cdot)\right]
$$

$\mathcal{P}^{i}$ denotes the price function perceived by agent i. It is used to extract information from the observed price. For the agent to be able to unveil the information conveyed by the price, he must guess on the equilibrium relationship between the price, $\nu$ and $\phi$. The next subsection describes in more detail how agents compute their beliefs in the class of economies we analyze.

A type i consumer solves the following optimization problem:

$$
\begin{aligned}
& \underset{c_{h}, c_{l}}{\operatorname{Max}}\left\{\tilde{\nu}^{i}\left(\mathcal{I}, p, \mathcal{P}^{i}(\cdot)\right) u\left(c_{h}\right)+\left(1-\tilde{\nu}^{i}\left(\mathcal{I}, p, \mathcal{P}^{i}(\cdot)\right)\right) u\left(c_{l}\right)\right\} \\
& \text { subject to } \quad(1-p) c_{l}+p c_{h}=W=a^{i}+(1-p) d_{l}+p d_{h} \\
& c_{l}, c_{h} \geq 0
\end{aligned}
$$

where: $u(c)$ denotes the utility function; $c_{j}$ denotes planned consumption in state $\mathrm{j} ; a^{i}$ denotes the riskless endowment of a type i agent; $d_{j}$ denote the dividends paid by the tree in state $\mathbf{j}$; and $W$ denotes individual wealth. The sum of the prices of the Arrow-Debreu securities is normalized to 1.

If each agent receives $n$ signals, there are $2(n+1)$ different types: an agent can receive either a high or low riskless endowment combined with $n+1$ different signals realizations. For simplicity, the paper assumes that the distribution of signals in the population is independent from the distribution of riskless endowments. Denote by $\mu^{i}(\nu, \phi, n)$ the measure of agents $\mathrm{i}$ in the population. For instance, the model implies there is a fraction $(1-\phi)\left(\begin{array}{l}n \\ j\end{array}\right) \nu^{j}(1-\nu)^{(n-j)}$ of agents with a low riskless endowment and $\mathrm{j}$ good signals.

Let $Y_{i}(\phi)$ denote the overall aggregate resources in state $\mathrm{i}, c_{j}^{i}\left(p, \mathcal{P}^{i}(\cdot)\right)$ denote the optimal consumption of agent $\mathrm{i}$ in state $\mathrm{j}$, and $Z_{i}\left(p, \mu, \mathcal{P}^{1}, \ldots, \mathcal{P}^{2(n+1)}\right)$ denote the aggregate demand in state $\mathrm{i}$. 
The latter is computed as follows:

$$
Z_{j}\left(p, \mu, \mathcal{P}^{1}(\cdot), \ldots, \mathcal{P}^{2(n+1)}(\cdot)\right)=\sum_{i=1}^{2(n+1)} c_{j}^{i}\left(p, \mathcal{P}^{i}(\cdot)\right) \mu^{i}(\nu, \phi, n) .
$$

We are now ready to define a competitive equilibrium for this class of economies.

Definition 1 A rational expectations equilibrium (REE) consists of a measurable price function $p$ : $\mathrm{I} \times \Phi \rightarrow[0,1]$ and individual demands $\left\{c_{l}^{i}(\cdot), c_{h}^{i}(\cdot)\right\}_{i=1}^{i=2(n+1)}$ such that:

(1) $\left\{c_{l}^{i}(p(\nu, \phi) p(\cdot)), c_{h}^{i}(p(\nu, \phi) p(\cdot))\right\}$ solve consumer i's problem $\forall i=1, \ldots, 2(n+1)$ and $\forall \nu \in \mathrm{I}, \phi \in \Phi$.

(2) Markets Clear: $Z_{j}(p(\nu, \phi), \mu(\nu, \phi), p(\cdot), \ldots, p(\cdot))=Y_{j} \forall j=l, h$ and $\nu \in \mathrm{I}, \phi \in \Phi$.

Radner (1979) provides a more general definition of the equilibrium concept defined above. ${ }^{7}$ An important assumption implicit in Definition 1 is that the individuals' perceived price function coincide with the actual equilibrium function. Agents, fully understand how prices are determined and take that into account to update their beliefs. Notice that in general, finding a solution of the previous problem requires solving for a fixed point functional equation: the price function perceived by the agents must coincide with the price function generated by their behavior.

In what follows we consider a simplified version of the framework described above. That reduces the generality of the results but allows us to characterize the equilibrium in some cases. Even though the conclusions depend on the specific assumptions we make, the economies analyzed do not belong to a negligible set, i.e. the results are robust to any perturbation of the primitives: utility function; dividends and endowment process; and joint distribution of $\nu$ and $\phi$.

\subsection{Finding the equilibrium: a particular case}

We choose a logarithmic utility function because it has the advantage that individual demands are linear in wealth. The optimal consumption rules are specified in equation (2). It is assumed for simplicity that

\footnotetext{
${ }^{7}$ Dubey et al. (1987) criticize the REE approach because it assumes implicitly that prices pool individuals' private information before they trade. Nonetheless, the approach has been extensively used in the literature, showing that, despite its limitations, it constitutes a useful tool to analyze problems with asymmetric information
} 
the random variables $\nu$ and $\phi$ are independent. $\nu$ is drawn from a uniform distribution with support $[0,1]$, while $\phi$ takes a high value $\phi_{h}$ with probability $\pi$ and a low value $\phi_{l}$ with probability $1-\pi$.

$$
c_{h}^{i}(p, p(\cdot))=\tilde{\nu}^{i}(p, p(\cdot)) \frac{W^{i}}{p} \quad c_{l}^{i}(p, p(\cdot))=\left(1-\tilde{\nu}^{i}(p, p(\cdot))\right) \frac{W^{i}}{1-p}
$$

In order to help to understand how the model works, we assume for the moment that each agent receives only one signal. This implies that there are four different types of agents in the model. They are listed below with their corresponding measure.

- $\nu \phi$ agents with high endowment and a good signal (denoted by $\overline{1}$ ),

- $(1-\nu) \phi$ agents with high endowment and a bad signal (denoted by $\overline{0})$,

- $\nu(1-\phi)$ agents with low endowment and a good signal (denoted by $\underline{1})$,

- $(1-\nu)(1-\phi)$ agents with low endowment and a bad signal (denoted by $\underline{0})$.

In equilibrium, aggregate planned consumption for the high state must equal aggregate resources in that state. If that equality holds, by Walras' law, the other market is also in equilibrium. The market clearing condition is formally stated in equation (3).

$$
\phi\left[\nu c_{h}^{\overline{1}}+(1-\nu) c_{h}^{\overline{0}}\right]+(1-\phi)\left[\nu c_{h}^{\underline{1}}+(1-\nu) c_{h}^{0}\right]=\phi \bar{a}+(1-\phi) \underline{a}+d_{l},
$$

where $\bar{a}$ denotes the high value of the riskless endowment and $\underline{a}$ its low value.

The equilibrium price is obtained after replacing individual demands into the market clearing condition.

$$
p(\nu, \phi)=\frac{\phi\left(\bar{a}+d_{l}\right)\left[\nu \tilde{\nu}^{\overline{1}}+(1-\nu) \tilde{\nu}^{\overline{0}}\right]+(1-\phi)\left(\underline{a}+d_{l}\right)\left[\nu \tilde{\nu}^{\underline{1}}+(1-\nu) \tilde{\nu}^{0}\right]}{\phi \bar{a}+(1-\phi) \underline{a}+d_{h}+\left\{\phi\left[\nu \tilde{\nu}^{\overline{1}}+(1-\nu) \tilde{\nu}^{\overline{0}}\right]+(1-\phi)\left[\nu \tilde{\nu}^{\underline{1}}+(1-\nu) \tilde{\nu}^{\underline{0}}\right]\right\}\left(d_{l}-d_{h}\right)}
$$

It is easy to show that this model does not possess a fully revealing equilibrium. The reasoning is as follows. The agents' private signals and endowments do not convey enough information to reveal the realization of $(\nu, \phi)$. Thus, the only way in which agents can infer the values of those variables is if in equilibrium there is a one to one mapping between $(\nu, \phi)$ and the equilibrium price. In other words, for 
prices to be fully revealing, there must be only one possible realization of $\nu$ consistent with a given price and value of $\phi$. The equilibrium relationship between $\nu$ and the last two variables in the fully revealing case is described in equation (5). ${ }^{8}$

$$
\nu(p, \phi)=\frac{p\left[\phi \bar{a}+(1-\phi) \underline{a}+d_{h}\right]}{\phi \bar{a}+(1-\phi) \underline{a}+(1-p) d_{l}+p d_{h}}
$$

It is apparent that there is more than one combination of $\nu$ and $\phi$ consistent with a given price. This contradicts the hypothesis that prices are fully revealing. Furthermore, it suggests that the equilibrium is pairwise revealing: the market price reveals that the probability of high dividends could have taken one of two possible values. Thus, individual beliefs consist on a weighted some of those values. The weights are determined by the signal and endowment received.

In order to understand how the price depends on $\nu$ and $\phi$, it is helpful to consider again the case where both variables are common knowledge. In that case, the equilibrium price function is given by

$$
p^{F R}(\nu, \phi)=\frac{\nu\left[\phi \bar{a}+(1-\phi) \underline{a}+d_{l}\right]}{\phi \bar{a}+(1-\phi) \underline{a}+d_{h}-\nu\left(d_{h}-d_{l}\right)} .
$$

The relative price trivially increases with $\nu$. As the high state becomes more likely, agents demand more contingent claims paying in that state. It can easily be shown that the equilibrium price also increases with $\phi$. We have already mentioned that 'poor' agents (with a low riskless endowment) are de-facto more risk averse than 'rich' agents, so the former ones buy insurance from the latter, i.e. agents with a low endowment transfer consumption from the high dividend state to the low dividend state. ${ }^{9}$ As the fraction of rich individuals $(\phi)$ increases, there are less agents demanding contingent claims that pay in the low state, so their relative price decreases, i.e., $p$ increases.

\footnotetext{
${ }^{8}$ Equation 5 is obtain after replacing individual beliefs $\tilde{\nu}^{i}$ in equation (4) by the actual realization of $\nu$.

${ }^{9}$ Let $\theta_{h}$ denote the net demand of contingent claims that pay only if the high state is realized. The agent is endowed with $a+d_{h}$ of this asset. It can be shown that$$
\frac{\partial \theta_{h}}{\partial a}>0 \Longleftrightarrow \frac{-u^{\prime \prime}\left(c_{h}\right)}{u^{\prime}\left(c_{h}\right)}<\frac{-u^{\prime \prime}\left(c_{l}\right)}{u^{\prime}\left(c_{l}\right)}
$$

where

$$
c_{i}=a+d_{i}+\theta_{i} \quad i=l . h
$$

From the individual first order conditions and the aggregate resource constraint, it transpires that $c_{h}>c_{l}$ for every agent. Thus, a sufficient condition for the previous inequality to hold is that the coefficient of absolute risk aversion decreases with consumption. The utility function assumed in the present paper satisfies this property.
} 
Presumably, the equilibrium price in the economy with asymmetric information is also increasing in both arguments. We found this to be true in all the simulations performed.

\subsubsection{Beliefs' updating scheme}

The equilibrium price specified in equation (4) takes the values of the beliefs as given. But, as it was explained before, the latter are a function of the market price and the price function itself. This section explains in more detail how agents compute their beliefs.

Figure 1 shows how agents extract the information pooled by the market price. Every agent is assumed to know the price function. So when they observe a particular price, say $p_{0}$ in the picture, they infer that only two values of $\nu$ could have been realized: $\nu\left(p_{0}, \phi_{l}\right)$ or $\nu\left(p_{0}, \phi_{h}\right)$. The first one corresponds to the value of $\nu$ consistent with a price $p_{0}$ and a low fraction of highly endowed agents. The second one corresponds to the value of $\nu$ consistent with a low fraction of highly endowed agents. Since agents ignore the actual distribution of riskless endowments, they cannot distinguish which of the previous values corresponds to the actual realization of $\nu$. But agents not only learn from the market price. Their private signals and endowments reveal information. An agent with a high endowment believes that it is more likely that the fraction of rich agents is $\phi_{h}$ rather than $\phi_{l}$, so he assigns more weight to $\nu\left(p_{0}, \phi_{h}\right)$. An agent with a good signal believes that it is more likely that the highest $\nu$ was realized.

We now formalize the previous argument taking the case of an agent who has received a high riskless endowment and a good signal. The beliefs' updating schemes of the remaining agents follow the same logic.

The paper assumes that each agent's belief regarding the probability that the tree pays high dividends consists of the expectation of $\nu$ conditional on his private information and the market price, namely

$$
\begin{aligned}
\tilde{\nu}^{\overline{1}}(p) & =E\left[\operatorname{Pr}\left(\text { tree pays } d_{h}\right) \mid \text { signal }=1 \text {, endowment }=\bar{a}, \text { price }=p\right] \\
& =\nu\left(p, \phi_{h}\right) \operatorname{Pr}\left(\nu\left(p, \phi_{h}\right) \mid 1, \bar{a}, p\right)+\nu\left(p, \phi_{l}\right) \operatorname{Pr}\left(\nu\left(p, \phi_{l}\right) \mid 1, \bar{a}, p\right) .
\end{aligned}
$$

The second equality takes into account that once the agent has conditioned on the price, the probability $\nu$ has a dichotomous distribution. Now, let us apply the law of conditional probabilities to the 


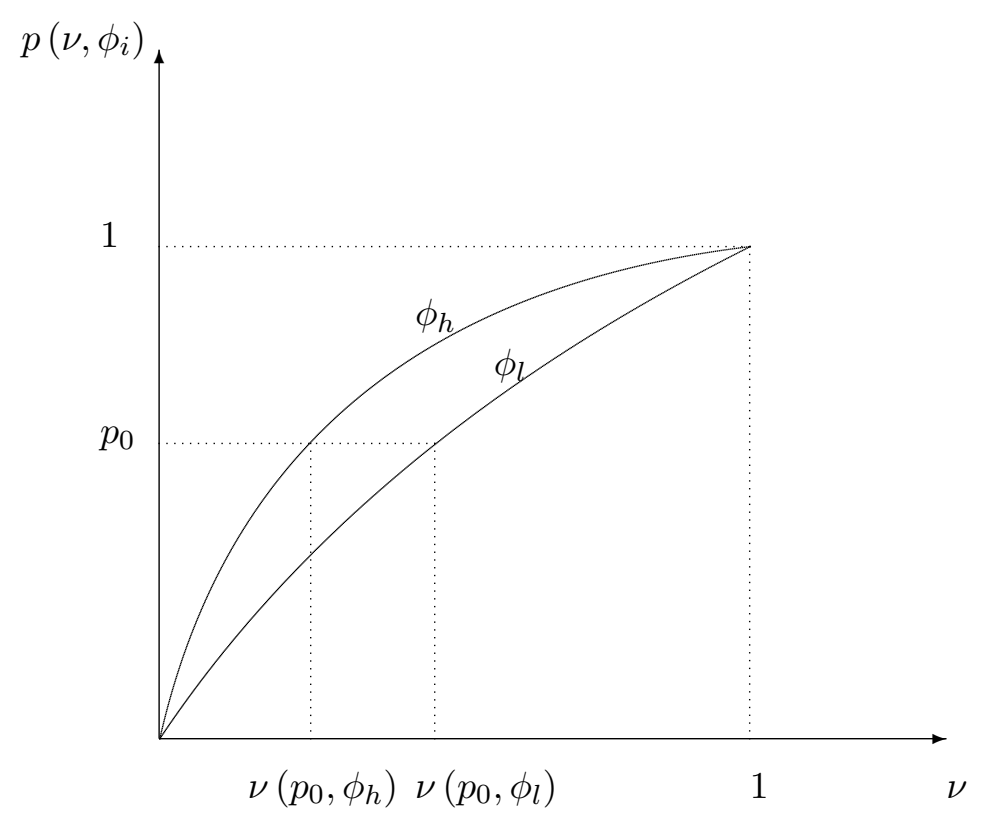

Figure 1: Information revealed by the price function

last expression, and then use the implication that once we condition on the fact that Nature has picked a particular value of $\phi$, the following events are mutually independent: the tree pays high dividends; the agent receives a good signal; and the agent receives a high riskless endowment. The result is the following equation:

$$
\tilde{\nu}^{\overline{1}}(p)=\frac{\left\{\begin{array}{c}
\nu\left(p, \phi_{h}\right) \operatorname{Pr}\left(1 \mid p, \phi_{h}\right) \operatorname{Pr}\left(\bar{a} \mid p, \phi_{h}\right) \operatorname{Pr}\left(p \mid \phi_{h}\right) \operatorname{Pr}\left(\phi_{h}\right)+ \\
\nu\left(p, \phi_{l}\right) \operatorname{Pr}\left(1 \mid p, \phi_{l}\right) \operatorname{Pr}\left(\bar{a} \mid p, \phi_{l}\right) \operatorname{Pr}\left(p \mid \phi_{l}\right) \operatorname{Pr}\left(\phi_{l}\right)
\end{array}\right\}}{\left\{\begin{array}{c}
\operatorname{Pr}\left(1 \mid p, \phi_{h}\right) \operatorname{Pr}\left(\bar{a} \mid p, \phi_{h}\right) \operatorname{Pr}\left(p \mid \phi_{h}\right) \operatorname{Pr}\left(\phi_{h}\right)+ \\
\operatorname{Pr}\left(1 \mid p, \phi_{l}\right) \operatorname{Pr}\left(\bar{a} \mid p, \phi_{l}\right) \operatorname{Pr}\left(p \mid \phi_{l}\right) \operatorname{Pr}\left(\phi_{l}\right)
\end{array}\right\}}
$$

Finally, equation (7) is obtained after replacing the probabilities in the last expression by their actual values. Recall that the probabilities of receiving a good signal and a high riskless endowment coincide with the actual realizations of $\nu$ and $\phi$, respectively.

$$
\tilde{\nu}^{\overline{1}}(p)=\frac{\nu\left(p, \phi_{h}\right) \nu\left(p, \phi_{h}\right) \phi_{h} g\left(p \mid \phi_{h}\right) \pi+\nu\left(p, \phi_{l}\right) \nu\left(p, \phi_{l}\right) \phi_{l} g\left(p \mid \phi_{l}\right)(1-\pi)}{\nu\left(p, \phi_{h}\right) \phi_{h} g\left(p \mid \phi_{h}\right) \pi+\nu\left(p, \phi_{l}\right) \phi_{l} g\left(p \mid \phi_{l}\right)(1-\pi)}
$$


The function $g\left(p \mid \phi_{i}\right)$ denotes the density of the price conditional on $\phi_{i}$, where

$$
g(p \mid \phi)=f\left(\nu\left(p, \phi_{i}\right)\right) \frac{\partial \nu\left(p, \phi_{i}\right)}{\partial p}=f\left(\nu_{i}(p)\right) \nu_{i}^{\prime}(p)
$$

The last equality just simplifies the notation. The subindex $i$ denotes the fraction of rich agents in the economy, i.e. $\phi_{i}$, and $f(\cdot)$ denotes the density function of $\nu$. ${ }^{10}$ The intuition for the formula of the conditional density is that a price $p$ is likely to be observed when the value of $\nu$ consistent with that price is likely to be drawn, i.e. $f(\nu)$ is high, or when the price function $p_{i}($.$) is not sensitive to \nu$ at $\nu_{i}(p)$. An heuristic description of the last argument is provided in the picture below. Consider an hypothetical case when it is known that the price lay on the range $\left[p_{0}, p_{1}\right]$. Its actual value, however, is not observed. In that case, agents infer that $\nu$ belong to $\left[\nu_{h}\left(p_{0}\right), \nu_{h}\left(p_{1}\right)\right]$ if the fraction of rich agents is $\phi_{h}$, and to $\left[\nu_{l}\left(p_{0}\right), \nu_{l}\left(p_{1}\right)\right]$ if the fraction is $\phi_{l}$. In the case where $\nu$ is drawn from a uniform distribution, the probability of observing a price in $\left[p_{0}, p_{1}\right]$ consists of the length of the interval $\left[\nu_{i}\left(p_{0}\right), \nu_{i}\left(p_{1}\right)\right]$, which is clearly higher for price function $p\left(\cdot, \phi_{l}\right)$. In the limit, as the length of the price range collapses to a single point, the likelihood of observing a particular price becomes inversely proportional to the derivative of the price function at that point, or directly proportional to $\nu_{i}^{\prime}(q)$.

The beliefs of the remaining types are described in $(8)-(10)$.

$$
\begin{aligned}
& \tilde{\nu}^{\overline{0}}(p)=\frac{\pi f\left(\nu_{h}\right) \nu_{h}^{\prime} \phi_{h}\left(1-\nu_{h}\right) \nu_{h}+(1-\pi) f\left(\nu_{l}\right) \nu_{l}^{\prime} \phi_{l}\left(1-\nu_{l}\right) \nu_{l}}{\pi f\left(\nu_{h}\right) \nu_{h}^{\prime} \phi_{h}\left(1-\nu_{h}\right)+(1-\pi) f\left(\nu_{l}\right) \nu_{l}^{\prime} \phi_{l}\left(1-\nu_{l}\right)} \\
& \tilde{\nu}^{\underline{1}}(p)=\frac{\pi f\left(\nu_{h}\right) \nu_{h}^{\prime}\left(1-\phi_{h}\right) \nu_{h}^{2}+(1-\pi) f\left(\nu_{l}\right) \nu_{l}^{\prime}\left(1-\phi_{l}\right) \nu_{l}^{2}}{\pi f\left(\nu_{h}\right) \nu_{h}^{\prime}\left(1-\phi_{h}\right) \nu_{h}+(1-\pi) f\left(\nu_{l}\right) \nu_{l}^{\prime}\left(1-\phi_{l}\right) \nu_{l}} \\
& \tilde{\nu}^{\underline{0}}(p)=\frac{\pi f\left(\nu_{h}\right) \nu_{h}^{\prime}\left(1-\phi_{h}\right)\left(1-\nu_{h}\right) \nu_{h}+(1-\pi) f\left(\nu_{l}\right) \nu_{l}^{\prime}\left(1-\phi_{l}\right)\left(1-\nu_{l}\right) \nu_{l}}{\pi f\left(\nu_{h}\right) \nu_{h}^{\prime}\left(1-\phi_{h}\right)\left(1-\nu_{h}\right)+(1-\pi) f\left(\nu_{l}\right) \nu_{l}^{\prime}\left(1-\phi_{l}\right)\left(1-\nu_{l}\right)}
\end{aligned}
$$

Notice that the equilibrium price affects the beliefs in two ways. First, for a given market price $p$, agents use the equilibrium price function to retrieve the possible realizations of $\nu: \nu_{h}(p)$ and $\nu_{l}(p)$. Second, they use the derivative of the price function $\left(\nu_{h}^{\prime}(p)\right.$ and $\left.\nu_{l}^{\prime}(p)\right)$ in order to assess how likely are those points.

\footnotetext{
${ }^{10}$ The paper assumes a uniform distribution over the interval $[0,1]$, so the density is just the constant 1 . However, it will assist the intuition to consider for the moment the more general case.
} 


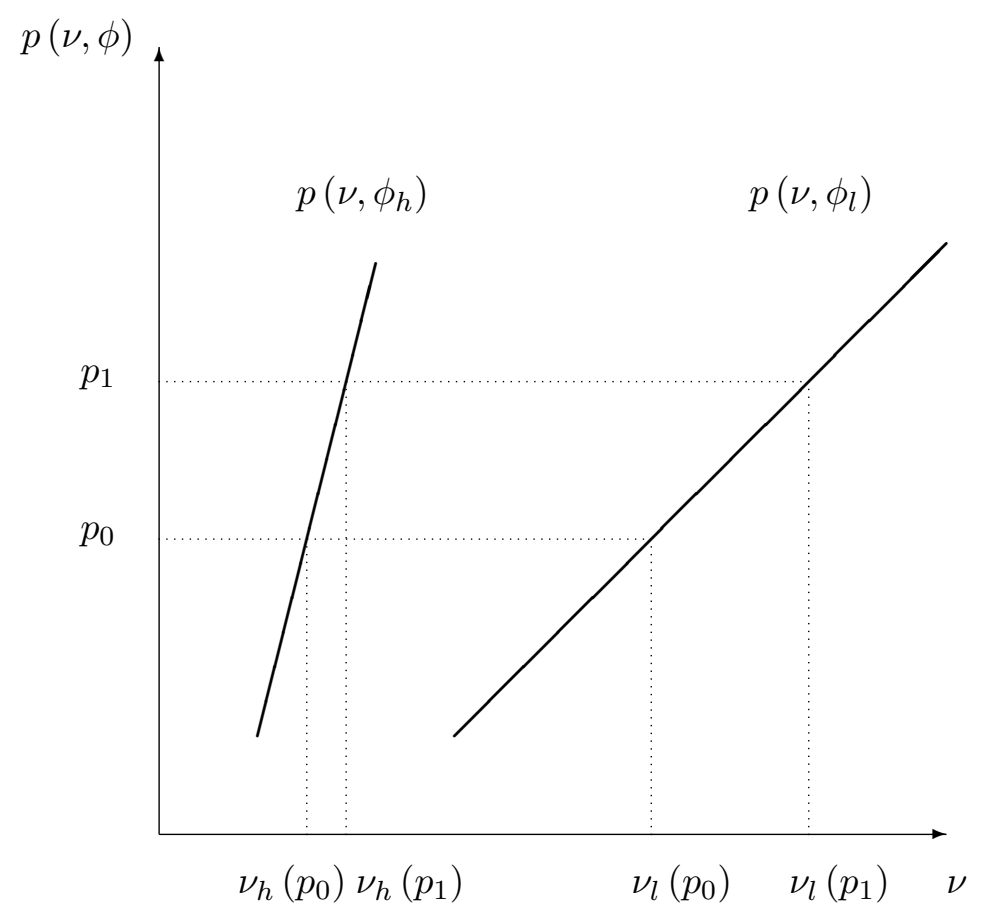

The structure of the model is similar to Ausubel (1990a) and Ausubel (1990b). He also analyzed an economy with partially revealing prices and where the state of the economy is characterized by two variables: one continuous and the other dichotomous. In our framework, the first one would be represented by $\nu$ and the second one by $\phi$. The difference is that he considered the case where a fraction of the population is fully informed while the rest is uninformed and must learn from the equilibrium price. This structure allowed him to prove existence and uniqueness of equilibrium. In Ausubel (1990a) he is also able to obtain a closed-form solution for the equilibrium price using specific assumptions about the utility function and the distribution of the continuous variable. Unfortunately, his results do not extend to the present framework. Our model does not allow for a tractable analytical solution. But an approximate solution can be found using numerical techniques. The appendix provides a detailed description of the procedure followed to find the equilibrium.

\section{Parametrization}

The model presented before builds on many restrictive assumptions. This allows us to find a (numerical) solution but has the disadvantage that the resulting model is highly stylized and has a limited ability 
to replicate real data. Thus, the parameters that characterize the dividends and endowment's processes are not chosen following a standard calibration exercise, i.e. they are not based on actual data. There are other reasons that motivate the previous choice. The fact of considering a risky asset that lives for only one period does not allow to mimic the returns of any aggregate stock index. ${ }^{11}$ Besides, in order to calibrate the process of the riskless endowment it would be necessary to consider not only the labor income of stockholders, but also other sources of income, like the returns to private businesses, which are not easy to obtain.

The strategy, therefore, is to choose a baseline parametrization that helps to illustrate the effect the paper tries to emphasize. To that end, the worst realization of the riskless endowment is allowed to take a relatively low value in order to magnify the different attitudes toward risk of rich and poor agents. This increases the sensitivity of the equilibrium to changes in the distribution of endowments (controlled by $\phi$ ). Similarly, if the dividend dispersion was low, equilibrium state prices would lay close to the corresponding state probability, regardless of the realization of $\phi$. In that case, agents' beliefs would tend to coincide with the actual realization of $\nu$, and the economy would behave almost as if everyone were fully informed. A disperse dividend realization is necessary to avoid that result. In summary, we restrict attention to the case where the lower realizations of the riskless endowment and dividend take small values compared to their higher counterpart. Both are necessary to generate significant belief's heterogeneity in the model. The parameters chosen are specified in the table below.

\begin{tabular}{|c|c|}
\hline$d_{h}=1$ & $d_{l}=0.1$ \\
\hline $\bar{a}=1$ & $\underline{a}=0.5$ \\
\hline$\phi_{h}=0.8$ & $\phi_{l}=0.2$ \\
\hline$\pi=0.5$ & $\nu \sim U(0,1)$ \\
\hline
\end{tabular}

\footnotetext{
${ }^{11}$ If the risk free bond is taken as numeraire, the expected return of the tree for a given realization of $\nu$ and $\phi$ in an economy with full information is

$$
R(\nu, \phi)=\frac{\nu d_{h} p}{d_{l}(1-p)+d_{h} p}+\frac{(1-\nu) d_{l}(1-p)}{d_{l}(1-p)+d_{h} p}, \quad \text { where } p=p(\nu, \phi) .
$$

The gross return is below 1 for almost all realizations of $\nu$ and $\phi$. This implies that the model cannot generate positive net rates of returns of the risky asset, as it is observed in the data.
} 


\section{Results}

Figure 2 compares the equilibrium prices between an economy with full information and an economy with asymmetric information (agents receive one signal). The graph shows that the monotonicity property of the price function is preserved in the asymmetric information framework. It also illustrates that when the economy is hit with a good endowment shock $\left(\phi=\phi_{h}\right)$, the relative price of the high dividend state is higher in the asymmetric information case compared to the full information case. The result is reversed when the economy is hit with a bad shock. The explanation rests on the beliefs' updating scheme. Consider again Figure 1 on page 14. Interpret the picture as the price scheme of the case where all the population but a single agent is fully informed. The unlucky agent has to infer $\nu$ from the price observed in the market and his private information. If the values $\nu\left(p_{0}, \phi_{l}\right)$ and $\phi_{l}$ are realized, the agent's belief lays below the actual realization of $\nu$. The equilibrium price is not affected because that single agent has measure zero and so his behavior does not influence aggregate variables. However, if the fraction of agents who are imperfectly informed increases, the average belief in the economy decreases and the equilibrium price falls, as can be deduced from equation (4). Eventually, if no agent is fully informed, the average believe is below the actual realization of $\nu$. This implies that the equilibrium price is below its level in the full information economy, as Figure 2 shows. The previous argument holds for any realization of $\nu$. A similar logic can be used to explain why the equilibrium price is higher in an economy with asymmetric information and a high realization of $\phi$.

Figures 3-4 graph the beliefs as a function of the price. It shows that the value of the riskless endowment conveys more information than the signal about the tree. Agents with low endowments are more optimistic than the rest, independently of the signal received. An agent hit with a low riskless endowment assigns more weight to the possibility that $\phi=\phi_{l}$ than a rich individual. This means that receiving a low endowment can be taken as a signal that the actual $\nu$ is closer to $\nu_{l}(p)$ than $\nu_{h}(p)$. The first value is higher than the second one, explaining why poor agents tend to be more optimistic.

The heterogeneity in beliefs along with the difference in the endowments induce agents to trade. In Section 2 we stated that in an economy with full information, rich agents sell contingent claims that pay in the low state. This may not be true in the present case. Poor agents are more optimistic than wealthy individuals, so the first ones may have now an incentive to transfer resources to the high state. 


\subsection{Definitions of welfare}

The objective of the paper is to assess, in a framework with asymmetric information, whether the receipt of more accurate public information increases or reduces individual welfare. But as Holmstrom and Myerson (1983) point out, the heterogeneity in private information raises a question that is not present in an economy with complete information: what is the appropriate measure of welfare? For instance, expected utility can be computed as a function of each agent's private information or it can be computed prior to the receipt of any private information. Holmstrom and Myerson denote the first concept as interim utility and the second one as ex ante utility. The paper employs the last two measures of welfare to present the results. Ex ante utility in the case where agents receive $\mathrm{n}$ signals is computed as follows:

$$
E(u)=\sum_{i=1}^{2} \sum_{j=0}^{n} \operatorname{Pr}\left(\phi_{i}\right) \int_{0}^{1}\left(\begin{array}{l}
n \\
j
\end{array}\right) \nu^{j}(1-\nu)^{(n-j)}\left[\phi_{i} u^{\bar{j}}\left(\nu, \phi_{i}\right)+\left(1-\phi_{i}\right) u^{\underline{j}}\left(\nu, \phi_{i}\right)\right] d \nu
$$

where $\mathcal{U}^{\bar{j}}(\nu, \phi)$ denotes the expected utility of an agent with $\mathrm{j}$ good signals and high riskless endowment, conditional on the information possessed at the node $(\nu, \phi)$. Similarly, $U^{\underline{j}}(\nu, \phi)$ denotes the conditional expected utility of an agent with low risk riskless endowment and $\mathrm{j}$ good signals. The conditional expected utility of an agent of type i is computed as follows:

$$
\mathcal{U}^{i}(\nu, \phi)=E\left[u \mid \mathcal{I}^{i}, p(\nu, \phi)\right]=\nu u\left(c_{h}^{i}(\nu, \phi)\right)+(1-\nu) u\left(c_{l}^{i}(\nu, \phi)\right)
$$

The decision rules that govern consumption in both states are the same as in equation (2) on page 11. Notice that even though agents are not able to observe $\nu$, the actual state probabilities are used to compute the expected utility of each type. It is easy to check that if those probabilities were replaced by agents' beliefs, the formula would yield the same level of ex ante utility. The formulation in equation (12) is chosen because it stresses that more precise information affects ex ante welfare only through its influence on the consumption allocation rules.

The model considered in this paper assumes there is no ex ante heterogeneity. Agents differ only after they have received endowments and signals. Thus, ex ante utility consists of a scalar variable. Even thought the previous measure is informative and allows us to evaluate aggregate welfare, it limits the ability to compare our results with the previous literature (that assumes exogenous heterogeneity). For 
that reason, we also provide a measure of interim welfare. The latter is computed after each individual has received his riskless endowment but prior to observing any signal or the market price, namely

$$
\begin{aligned}
E(u \mid a) & =\sum_{i=1}^{2} \operatorname{Pr}\left(\phi_{i} \mid a\right) E\left[u \mid a, \phi_{i}\right], \quad \text { where } \\
E\left[u \mid \bar{a}, \phi_{i}\right] & =\sum_{i=0}^{n} \int_{0}^{1}\left(\begin{array}{c}
n \\
i
\end{array}\right) \nu^{i}(1-\nu)^{(n-i)} \mathcal{U}^{\bar{i}}\left(\nu, \phi_{i}\right) d \nu, \\
E\left[u \mid \underline{a}, \phi_{i}\right] & =\sum_{i=0}^{n} \int_{0}^{1}\left(\begin{array}{c}
n \\
i
\end{array}\right) \nu^{i}(1-\nu)^{(n-i)} \mathcal{U}^{i}\left(\nu, \phi_{i}\right) d \nu .
\end{aligned}
$$

$\operatorname{Pr}\left(\phi_{i} \mid a\right)$ is computed using Bayes' rule.

The formula above assumes that agents update the probability distribution of $\phi$ before computing their expected utility. The reason is twofold. First, it is consistent with the rest of the paper, i.e. agents are fully rational and use all the available information when they evaluate their expected utility. Second, and also related to the previous reason, it is consistent with the ex ante utility measure. If the unconditional probability distribution of $\phi$ had been used in equation (13), it would not have been possible to relate both welfare measures.

For the sake of simplicity, the previous welfare measures were computed under the assumption that agents receive only one signal. They can easily be generalized to the multiple signal case.

\subsection{Welfare and the precision of information}

Appendix B considers a simpler version of the model, where agents learn only from public signals. It shows that as the number signals increases, information becomes more precise in Blackwell's sense (see Blackwell (1953)). The information structure assumed in this paper is different, though. Instead of learning from a public signal, agents learn from the market price and private information. This leads to a richer model but has the disadvantage that it is no longer possible to apply the standard definition of better information used in the literature. The result in Appendix B may support the conjecture that the precision of the information increases with the number of signals. In order to compare two information structures, then, we provide a measure of how 'far' are the beliefs with respect to the actual realizations of $\nu$. As it is described at the end of the section, that measure confirms the presumption that receiving 
more signals implies more accurate information.

We are now ready to present the main result of the paper. Figure 5 and Figure 6 show that welfare may be nonmonotonic in the precision of information. The relationship between welfare and the number of signals is described by a reverse $\mathrm{J}$ curve. This is independent of individual endowments, implying that ex ante welfare follows the same pattern.

The values at the boundaries of the graphs correspond to the null and complete information cases. It is not surprising that expected utility under full information is lower than under no information. The reason is the following. Uncertainty about $\nu$ and $\phi$ introduces an extra source of risk in the economy. Some agents would like to insure against 'bad' realizations of those variables, while others could gain from selling insurance. We consider economies where that market is missing. Agents trade only after observing their private information and market price. Given the previous restriction, the best allocation is attained when agents trade knowing their riskless endowment but with no information about $\nu .{ }^{12}$ In that economy, even if there was another trading round after the value of $\nu$ has been disclosed, there would be no further net trade. ${ }^{13}$ Prices would adjust in order to accommodate to the new 'belief', but the consumption allocation would remain invariant. This result was first pointed out in Marshall (1974). On the other hand, if markets open after the value of $\nu$ has become common knowledge, there are less opportunities to share risks. For instance, if $\nu$ takes an extreme value (zero or one), one of the Arrow-Debreu securities is valueless and no trade takes place. The example illustrates a general principle: the better the information agents possess, the lower the possibility to insure against 'bad news'. This is known as the Hirshleifer effect.

Blackwell (1953) was the first to formalize the intuitive result that more information is welfare enhancing. In his framework, agents receive an informative signal about the state and then choose an action $a$ out of a set $\mathcal{A}$. He shows that, as the signal transmits more accurate information, agents enjoy a higher degree of freedom when decide the optimal action. In the event that the signal is fully informative, agents can condition their decision on the actual state. Blackwell's result, though, relies on the fact that the set $\mathcal{A}$ does not change with the precision of information. Hirshleifer (1971) shows that this assumption cannot be maintained in a general equilibrium model. In a competitive environment,

\footnotetext{
${ }^{12}$ The paper restricts attention to economies where agents cannot insure against endowment shocks.

${ }^{13}$ The endowment allocation prior to the second trading round consists of the equilibrium allocation obtained in the first trading round.
} 
the arrival of more precise information about the state of the economy affects the equilibrium prices and through that, it modifies each individual's budget constraint. In fact, as it is stated in the previous paragraph, better information limits the opportunities to share risks, which in turn offsets Blackwell effect.

Several authors have studied the implications of Hirshleifer effect in more general environments. More recently, Schlee (2001) provides quite general conditions under which the receipt of better information leads to a Pareto inferior allocation, meaning that no agent is better off, and at least some agent is worse off (as long as the arrival of more precise information modifies the consumption allocation). Unlike previous papers, Schlee's conclusion does not depend on any specific assumption about agents' initial level of information: his result holds for any informational improvement, and not only when agents receive partial information starting from no information.

Figures 5 and 6 point out that there exist endowment economies with competitive markets in which individual welfare may increase with the precision of information. The graphs show that both types of agents enjoy higher expected utility under full information compared to the situation when they receive one signal. The Hirshleifer effect is still present in our model, but the asymmetric information assumption introduces an extra adverse effect on trade that is not observed in Schlee's paper.

The dispersion of beliefs is such that decreases agents' needs to participate in the market. In an equilibrium with homogeneous beliefs, individuals with a high riskless endowment provide insurance to poor agents by selling risk free bonds and purchasing shares of the tree. In the economy we study, however, there is disagreement in the beliefs about $\nu$. Rich agents are more pessimistic than poor agents. This feature is summarized in Figure 8. The picture shows that for any realization of $\nu$, poor agents' average beliefs are above the actual $\nu$, while rich agents' average beliefs are below $\nu$. This induces a contraction in the demand and supply of insurance compared to the full information setting. Thus, an economy with partial information displays a lower magnitude of trade than the economy where $\nu$ is common knowledge. The latter accounts for the difference in welfare between those two cases.

More generally, Figures 5 and 6 summarize the interaction between two effects. At the corners we observe the pure Hirshleifer effect, while at the 'interior' points the negative effect caused by the heterogeneity in beliefs plays a role. Both forces limit the opportunities to share risks. That explains why the arrival of better information always reduces welfare if agents have initially no information. However, 
the consequences of receiving better information starting from a situation of partial information are ambiguous. The receipt of more signals strengthens the Hirshleifer effect at the same time that it weakens the heterogeneous beliefs effect (agents' beliefs tend to concentrate). If the latter outweighs the former effect, we should observe a welfare increase following the arrival of more precise information. This is the situation described in the graph.

If the argument described in the previous paragraph is correct, we should expect welfare and trading volumes to be strongly correlated with each other. Figure 7 confirms this feature. The average volume of trade displays the same reverse $\mathrm{J}$ curve as welfare.

We cannot use Blackwell's criterion to compare the degree of precision between different information structures, so we are not able to assess a priori whether receiving more signals implies better information or not. As it may be expected, though, there is a positive ex post relationship between the number of signals received and the accuracy of the beliefs. This is confirmed by Figure 8, which shows that the distance between individual beliefs and the actual realizations of $\nu$ shrinks as the number of signals increases. In addition, this result seems to hold for every possible value of $\nu$ and $\phi$. Figure 9 uses a simple measure to quantify the precision of the beliefs. ${ }^{14}$ The picture also supports the conjecture that more signals imply better information.

\subsection{Incentives to acquire information}

We have shown how the receipt of informative signals can hurt everyone in the economy. But this result relies on the assumption that agents actually use all the available information to compute their beliefs. The assumption should not be maintained if each individual finds that it is in his own interest to ignore all or part of the information received. We presume this is not the case in our model. Recall that no agent can exert any influence on the aggregate. This implies that, from the point of view of a single agent, the arrival of better information has a pure Blackwell effect, so it should be welfare enhancing. The chart below illustrates the private incentives to use information in a particular case. It describes

\footnotetext{
${ }^{14}$ The graph summarizes the average distance between individual beliefs and realizations of $\nu$ using the following measure:

$$
\left.\left.\sum_{i=1}^{2} \sum_{j=0}^{n} \operatorname{Pr}\left(\phi_{i}\right) \int_{0}^{1} \begin{array}{c}
n \\
j
\end{array}\right) \nu^{j}(1-\nu)^{n-j} \quad \phi_{i} \tilde{\nu}^{\bar{j}}-\nu^{2}+\left(1-\phi_{i}\right) \quad \tilde{\nu}^{j}-\nu^{2} d \nu\right)^{\frac{1}{2}}
$$
}


the expected utility of an individual who lives in our benchmark economy: the set of agents who use all the information available to update their beliefs has full measure. Besides, agents receive only one signal. It's clear that every other choice, but to use the information contained in the price, the signal and the endowment realization, leads to a worse decision rule.

\begin{tabular}{|l|c|c|}
\hline \multicolumn{3}{|c|}{ Individual Welfare when different } \\
pieces of information are used 15 \\
\hline Price, signals and endowment & Rich & Poor \\
Price and endowment only & 100.0000 & 100.0000 \\
Price and signals only & 99.9966 & 99.9966 \\
Price only & 99.8008 & 99.8008 \\
Signal only & 99.7932 & 99.7931 \\
No information & 87.5619 & 87.5619 \\
\hline
\end{tabular}

Similarly, consider an economy where no one uses any information. In that case, agents' beliefs are homogeneous and coincide with the unconditional expectation of $\nu$. But if an individual decides to incorporate the information revealed by the signal to update his belief, he will enjoy an increase in welfare (expressed in terms of consumption) of $5.98 \%$ or $6.11 \%$ depending on whether he has received a high or low riskless endowment, respectively.

Even though the complexity of the model does not allow us to prove the conjecture that every individual has a private incentive to use all the available information, we could show that this is true for the baseline parametrization. The result has an additional implication. If agents were required to pay for the signals and that cost were sufficiently low, then every individual would effectively acquire a signal. 16 But that would lead to an overacquisition of information due to the Hirshleifer and heterogeneous beliefs effects. A similar result is reported in Berk (1997) using a model with strategic behavior.

\footnotetext{
${ }^{15}$ Welfare is expressed in terms of certainty equivalent consumption. The latter is normalized to 100 in the case where the individual uses all the information available.

${ }^{16}$ For simplicity, we assume at the moment that agents can only purchase one signal.
} 


\section{Concluding remarks}

Hirshleifer was the first to point out that in a general equilibrium framework, better information may leave some agents worse off. Although he considered a simple example, his conclusion has proven to be robust to several generalizations. In a recent paper, Schlee analyzed a general class of economies and concluded that better information reduces the expected utility of every agent.

The present work stresses that those results depend crucially on the existence of homogeneous beliefs. We consider an economy with endogenous heterogeneity, asymmetric information and partially revealing prices. The paper identifies two effects through which information affects the equilibrium allocation: the well known Hirshleifer effect; and an adverse effect induced by the heterogeneity in beliefs. From an ex ante perspective, both effects limit the possibilities to share risks and therefore, have a negative impact on welfare. In this setup, the arrival of more precise information strengthens the Hirshleifer effect at the same time that it weakens the heterogenous beliefs effect. It is not possible to establish a priori which effect dominates, raising the possibility that welfare might be nonmonotonic in the precision of information. The paper focuses on the last case. It analyzes an example where the impact of better information on welfare depends on the initial level of information: more precise information increases welfare in an economy with partial information; but it decreases welfare in an economy with no information.

To the best of our knowledge, the mechanism explaining the result has not been explored in the literature. The closest antecedent of this work is Citanna and Villanacci (2000). They also consider an economy with asymmetric information and partially revealing prices. Like this paper, they conclude that welfare may increase after the arrival of more accurate information. But they argue that the result is explained by wealth effects due to changes in relative prices (they study a multiple good economy).

The policy implications derived from the present work also differ from the previous literature. If the first best cannot be implemented (agents trade prior to the arrival of information), it might be possible to find policies that lead to a Pareto superior allocation. Those policies must induce agents to acquire more information.

From a security design perspective, the example studied in the paper favors a complete market structure. Introducing a new security in a partial information economy would complete the markets 
and allow agents to fully infer the value of $\nu$. In that case, the second best allocation would be attained. However, if we depart from the benchmark setup, it may be possible to get back the inverse relationship between welfare and the precision of information. ${ }^{17}$ In those economies, an incomplete financial structure is optimal. 18

\footnotetext{
${ }^{17}$ That kind of relationship would be observed in economies where the dispersion of beliefs is not as strong as in our example.

${ }^{18}$ See Marín and Rahi (2000) for more on this topic.
} 


\section{References}

Admati, A. (1985). 'A Noisy Rational Expectations Equilibrium for Multy-Asset Securities Markets'. Econometrica, volume 53, no. 3, 629-658.

Ausubel, L. M. (1990a). 'Insider Trading in a Rational Expectations Economy'. American Economic Review, volume 80, no. 5, 1023-41.

Ausubel, L. M. (1990b). 'Partially-Revealing Rational Expectations Equilibrium in a Competitive Economy'. Journal of Economic Theory, volume 50, 93-126.

Banerjee, A. and Seccia, G. (2002). 'On the 'Hirshleifer effect' of Unscheduled Monetary Policy Announcements'. Discussion Papers in Economics and Econometrics, University of Southampton.

Berk, J. B. (1997). 'The Acquisition of Information in a Dynamic Market'. Economic Theory, volume 9, $441-451$.

Bhattacharya, U. and Matthew, S. (1991). 'Insiders, Ousiders, and Market Breakdown'. The Review of Financial Studies, volume 4, no. 2, 255-282.

Blackwell, D. (1953). 'Equivalent Comparisons of Experiments'. The Annals of Mathematical Statistics, volume 24 , no. 2, 265-272.

Citanna, A. and Villanacci, A. (2000). 'Incomplete Markets, Allocative Efficiency, and the Information Revealed by Prices'. Journal of Economic Theory, volume 90, 222-253.

Diamond, D. and Verrecchia, R. (1981). 'Information Aggregation in a Noisy Rational Expectations Economy'. Journal of Financial Economics, volume 9, 221-235.

Drees, B. and Eckwert, B. (2003). 'Welfare Effects of Transparency in Foreign Exchange Markets: the Role of Hedging Opportunities'. Review of International Economics, volume 11, no. 3, 453-463.

Drèze, J. H. (1960). 'Le paradoxe de l'information'. Economie appliquée, volume 13, 71-80. 
Dubey, P., Geanakoplos, J., and Shubik, M. (1987). 'The Revelation of Information in Strategic Market Games, A Critique of Rational Expectations Equilibrium'. Journal of Mathematical Economics, volume 16, 105-137.

Eckwert, B. and Zilcha, I. (2003). 'Incomplete Risk Sharing Arrangements and the Value of Information'. Economic Theory, volume 21, 43-58.

Gottardi, P. and Rahi, R. (2001). 'Efficiency Properties of Rational Expectations Equilibria with Asymmetric Information'. Centre for Economic Policy Research, Discussion Paper Series.

Green, J. (1981). 'Value of Information with Sequential Futures Markets'. Econometrica, volume 49, no. $2,335-358$.

Grossman, S. (1976). 'On the Efficiency of Competitive Stock Markets Where Traders Have Diverse Information'. The Journal of Finance, volume 31, no. 2, 573-585.

Grossman, S. and Stiglitz, J. (1980). 'On the Imposibility of Informationally Efficient Markets'. American Economic Review, volume 70, no. 3, 393-408.

Hakansson, N., Kunkel, G., and Ohlson, J. (1982). 'Sufficient and Necessary Conditions for Information to have Social Value in Pure Exchange'. Journal of Finance, volume 37, no. 5, 1169-1181.

Hatchondo, J., Krusell, P., and Schneider, M. (2003). 'Equilibrium Asset Trading with Incompletely Revealed Information'. Working Paper.

Hellwig, M. (1980). 'On the Aggregation of Information in Competitive Markets'. Journal of Economic Theory, volume 22, 477-498.

Hirshleifer, J. (1971). 'The Private and Social Value of Information and the Reward to Inventive Activity'. American Economic Review, volume 61, no. 4, 561-574.

Holmstrom, B. and Myerson, R. B. (1983). 'Efficient and Durable Decision Rules with Incomplete Information'. Econometrica, volume 51, no. 6, 1799-1820.

Judd, K. L. (1998). Numerical Methods in Economics. MIT. 
Lerman, C., Narod, S., Schulman, K., and Hughes, C. (1996). 'BRCA1 Testing in Families with Hereditary Breast-Ovarian Cancer'. Journal of the American Medical Association, volume 275, no. 24, 1885-92.

Marín, J. and Rahi, R. (2000). 'Information Revelation and Market Incompleteness'. Review of Economic Studies, volume 67, 455-481.

Marshall, J. (1974). 'Private Incentives and Public Information'. American Economic Review, volume 64 , no. $3,373-390$.

Ng, D. (1975). 'Information Accuracy and Social Welfare under Homogeneous Beliefs'. Journal of Financial Economics, volume 2, 53-70.

Quaid, K. and Morris, M. (1993). 'Reluctance to Undergo Predictive Testing for Huntington's Disease'. American Journal of Medical Genetics, volume 45, no. 1, 41-45.

Radner, R. (1979). 'Rational Expectations Equilibrium: Generic Existence and the Information Revealed by Prices'. Econometrica, volume 47, no. 3, 655-678.

Rahi, R. (1996). 'Adverse Selection and Security Design'. Review of Economic Studies, volume 63, 287-300.

Schlee, E. (2001). 'The Value of Information in Efficient Risk-Sharing Arrangements'. American Economic Review, volume 91, no. 3, 509-524.

Sulganik, E. and Zilcha, I. (1996). 'The Value of Information in the Presence of Future Markets'. The Journal of Future Markets, volume 16, no. 2, 227-240.

Verrecchia, R. (1982). 'Information Acquisition in a Noisy Rational Expectation Economy'. Econometrica, volume 50, no. 6, 1415-1430. 


\section{A Technical Appendix}

In order to study different properties of the equilibrium, we must find a price function $p(\nu, \phi)$ that satisfies certain conditions. The equilibrium is defined then by a particular relationship between the price, $\nu$ and $\phi$, which means that any of these variables can be expressed as a function of the other ones. It simplifies the exposition to write $\nu$ as a function of $p$ and $\phi$, so we proceed that way. If agents receive only one signal, the function $\nu(\cdot)$ satisfies equation (A.1). The formula follows from the equilibrium price equation (4) on page 11. It also takes the beliefs as exogenously given.

$$
\begin{aligned}
\nu_{i}(p) & =\nu\left(p, \phi_{i}\right)=\frac{p\left[\phi \bar{a}+(1-\phi) \underline{a}+d_{h}\right]-\left[\phi \bar{W} \tilde{\nu}^{\overline{0}}+(1-\phi) \underline{W} \tilde{\nu}^{\underline{0}}\right]}{\phi \bar{W}\left(\tilde{\nu}^{\overline{1}}-\tilde{\nu}^{\overline{0}}\right)+(1-\phi) \underline{W}\left(\tilde{\nu}^{1}-\tilde{\nu}^{\underline{0}}\right)} \\
\underline{\mathrm{W}} & =\underline{a}+d_{l}(1-p)+d_{h} p \\
\bar{W} & =\bar{a}+d_{l}(1-p)+d_{h} p
\end{aligned}
$$

More generally, if agents receive $\mathrm{n}$ signals, the equilibrium price is given by

$$
p(\nu, \phi)=\frac{\phi\left(\bar{a}+d_{l}\right) \sum_{h=0}^{n}\left(\begin{array}{l}
n \\
h
\end{array}\right) \nu^{h}(1-\nu)^{n-h} \tilde{\nu}^{\bar{h}}+(1-\phi)\left(\underline{a}+d_{l}\right) \sum_{h=0}^{n}\left(\begin{array}{l}
n \\
h
\end{array}\right) \nu^{h}(1-\nu)^{n-h} \tilde{\nu}^{\underline{h}}}{\phi \bar{a}+(1-\phi) \underline{a}+d_{h}-\left(\sum_{h=0}^{n}\left(\begin{array}{l}
n \\
h
\end{array}\right) \nu^{h}(1-\nu)^{n-h} \tilde{\nu}^{\bar{h}}+\sum_{h=0}^{n}\left(\begin{array}{l}
n \\
h
\end{array}\right) \nu^{h}(1-\nu)^{n-h} \tilde{\nu}^{\underline{h}}\right)\left(d_{h}-d_{l}\right)^{2}},
$$

which is nonlinear in $\nu$. The latter precludes the possibility of obtaining a functional expression for $\nu(\cdot)$, though it is possible to approximate the last function using numerical techniques.

Before describing the numerical algorithm, notice that the object that needs to be found (the function $\nu(\cdot))$ has infinite dimension, whereas numerical techniques only allow to solve for finite dimensional problems. Our strategy consists of parameterizing $\nu(\cdot)$ as the weighted sum of Chebychev polynomials. Namely,

$$
\begin{aligned}
& \nu_{j}(p) \simeq \hat{\nu}\left(p ; \vec{a}_{j}\right)=\sum_{i=0}^{i=N} a_{i}^{j} T_{i}\left(2 \frac{p-\underline{p}}{\bar{p}-\underline{p}}-1\right) \\
& \nu_{j}^{\prime}(p) \simeq \hat{\nu}^{\prime}\left(p ; \vec{a}_{j}\right)=\sum_{i=0}^{i=N} a_{i}^{j} T_{i}^{\prime}\left(2 \frac{p-\underline{p}}{\bar{p}-\underline{p}}-1\right)\left(\frac{2}{\bar{p}-\underline{p}}\right) \\
& \text { for } j=l, h
\end{aligned}
$$


where $\vec{a}_{h}=\left(a_{h}^{0}, a_{h}^{1}, \ldots, a_{h}^{N}\right), \vec{a}_{l}=\left(a_{l}^{0}, a_{l}^{1}, \ldots, a_{l}^{N}\right)$ are the corresponding weights and $T_{i}(\cdot)$ is the Chebychev polynomial of order i.

$$
T_{n}(x)=\cos \left(n \cos ^{-1} x\right)
$$

With this approach, the choice of the polynomial family becomes an important issue. We use Chebychev polynomials because they are mutually orthogonal and allow for an efficient parametrization of $\nu(\cdot)$. Finally, notice that the problem simplifies now to finding a finite number of parameters, instead of an entire function. The algorithm that solves the problem is laid down below.

1) A grid for $p$ is defined using the expanded Chebychev array (see Judd (1998), page 222). $\vec{p}=\left(p_{0}, p_{1}, \ldots, p_{N}\right)$, where $p_{0}=0$ and $p_{N}=1$. The grid defines the points at which the functions $\nu(\cdot)$ and $\hat{\nu}(\cdot)$ are evaluated.

2) A closed form solution exists in the full information economy. (see equation (5) on page 12) Thus, a first set of values for the parameters $\vec{a}_{h}$ and $\vec{a}_{l}$ is obtained after equating the approximate function $\hat{\nu}\left(p ; \vec{a}_{j}\right)$ to the known function $\nu^{F R}(\cdot)$ at the $N$ grid points. This gives $2 N$ equations in $2 N$ unknowns.

3) The values $\left\{a_{i}^{h}, a_{i}^{l}\right\}_{i=1}^{i=N}$ found in 2) are then used as an initial guess to solve for the system of equations defined by $G(\cdot)$.

$$
G_{i j}\left(\vec{a}_{h}, \vec{a}_{l}\right)=\nu_{j}\left(\hat{\nu}\left(p_{i} ; \vec{a}_{h}\right), \hat{\nu}\left(p_{i} ; \vec{a}_{l}\right), \hat{\nu}^{\prime}\left(p_{i} ; \vec{a}_{h}\right), \hat{\nu}^{\prime}\left(p_{i} ; \vec{a}_{l}\right), p_{i}\right)-\hat{\nu}\left(p_{i} ; \vec{a}^{j}\right), \quad i=0,1, \ldots, N, \quad j=l, h
$$

The function $\nu_{i}\left(\nu_{h}, \nu_{l}, \nu_{h}^{\prime}, \nu_{l}^{\prime}, p\right)$ refers to the same object as equation (A.1), but the arguments are different. The new formulation does not take the beliefs as exogenously determined. Instead, it includes as additional arguments the equilibrium values of $\nu$ consistent to a price $p$ (and the two possible realizations of $\phi$ ) and the derivatives of the price function, captured by $\nu_{i}^{\prime}$. The last four variables are necessary to compute the beliefs, as can be seen in equations (7)-(10).

The system of equations (A.2) summarizes the fixed point problem. The function $G_{i}^{j}(\cdot)$ does the following. It takes the set of parameters that defines $\hat{\nu}_{h}(\cdot)$ and $\hat{\nu}_{l}(\cdot)$ as arguments. Then, it computes $\nu_{h}\left(p_{i}\right), \nu_{l}\left(p_{i}\right)$ and their derivatives by evaluating the corresponding parameterized functions $\hat{\nu}\left(p ; \vec{a}_{j}\right)$ and $\hat{\nu}^{\prime}\left(p ; \vec{a}_{j}\right)$ at each price $p_{i}$ in the grid. If the approximate functions $\hat{\nu}(\cdot)$ are sufficiently close to 
the actual equilibrium functions, the evaluation of $\nu_{j}\left(., p_{i}\right)$ at the values described in the last sentence should yield a number similar to $\hat{\nu}\left(p_{i} ; \vec{a}^{j}\right): G_{i}^{j}(\cdot)$ should be close to zero. Therefore, the purpose of the algorithm is to find the root of the system of equations defined above.

The root is found using a modified Powell hybrid algorithm and a finite-difference approximation to the Jacobian. This corresponds to routine NEQNF of the IMSL library. A root is defined as a set of parameters $\left\{a_{i}^{j}\right\}_{i=l, h \quad j=0,1, \ldots, N}$ such that

$$
\sum_{i=l, h} \sum_{j=0}^{N} G_{i}^{j}\left(\vec{a}_{h}, \vec{a}_{l}\right)^{2}<10^{-12}
$$

The solution was tested at prices outside the Chebychev nodes defined for $q$. The average value of $\left|G_{i}^{j}\left(\vec{a}_{h}, \vec{a}_{l}\right)\right|$ at these prices is never larger than $10^{-5}$. The maximum deviation observed is below $10^{-3}$ and is always located at prices close to the corners. The latter suggests the numerical solution we obtain is very close to the actual one. 


\section{B Comparing information structures}

Let us introduce the following modifications to the baseline model presented in the paper:

1. the signals about the tree are public;

2. there is no uncertainty regarding the distribution of riskless endowments, i.e. the fraction of highly endowed agents is common knowledge.

Given the previous assumptions, the equilibrium price does not reveal more information than what the public signals does. The other difference with respect to the model considered in the main text, is that the economy is now characterized by the realization of one variable: $\nu$. Agents try to infer the latter using the information contained in the public signal. More formally, we assume agents observe a

signal $s$ from a set $\mathcal{S}$. The latter has finitely many components: $\mathcal{S}=\left\{s_{1}, s_{2}, \ldots\right\}$. The components of $\mathcal{S}$ are defined as follows:

$s_{1}=1$, agents observe one good signal out of one;

$s_{2}=0$, agents observe one good signal out of one;

$s_{3}=(1,1)$, agents observe two good signals out of two;

$s_{4}=(1,0)$, agents observe one good and bad signal out of two;

$s_{5}=(0,0)$, agents observe two bad signals out of two;

$s_{6}=(1,1,1$,$) , agents observe three good signals out of three;$

$\vdots$

Thus, each $s_{i}$ corresponds to a particular realization of the binary signals structure. The conditional probability distribution over $\mathcal{S}$ is given by a vector $\pi^{n}(\nu)=\left(\pi_{1}^{n}(\nu), \pi_{2}^{n}(\nu), \ldots\right)$, where

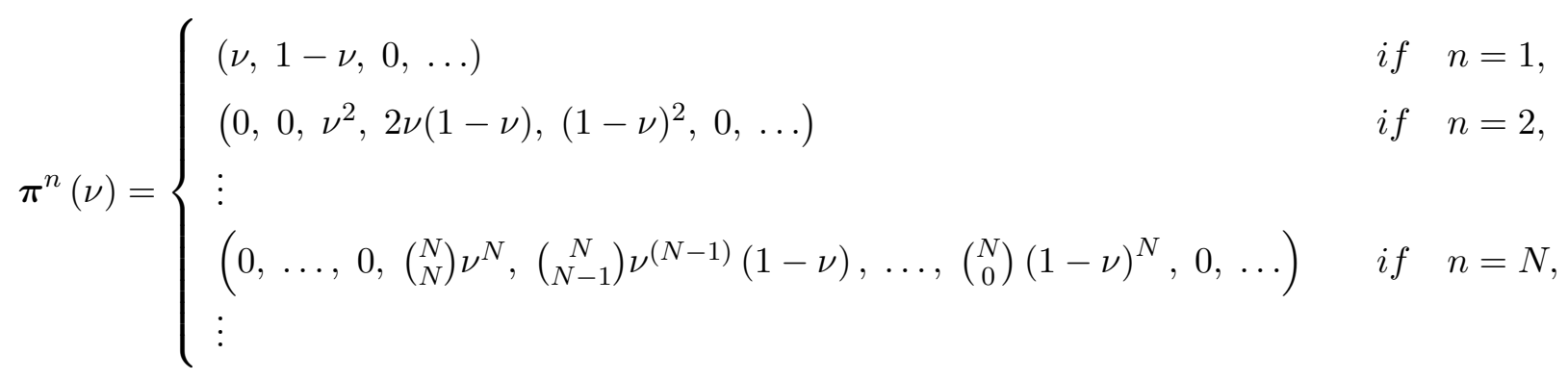

An information structure consists then of a collection of signals and their corresponding probabilities for every possible realization of $\nu$. In the present framework, an information structure is 
fully specified by the number of binary signals agents observe $(n)$, and it is denoted by $\mathbf{I}^{n}$, where $\mathbf{I}^{n}=\left\{\mathcal{S}, \boldsymbol{\pi}^{n}(\nu)\right.$ for $\left.\nu \in[0,1]\right\}$. The advantage of this set up is that it allows us to apply Blackwell's criterion to compare the degree of informativeness of two information structures.

Definition B.1 Information structure $\mathbf{I}^{n}$ is more informative than $\mathbf{I}^{n^{\prime}}$ if there is a transition probability function $t\left(s^{\prime}, s\right): \mathcal{S} \times \mathcal{S} \rightarrow[0,1]$, such that $\sum_{j=1}^{\infty} t\left(s_{j}, s\right)=1 \forall s \in \mathcal{S}$ and $\pi_{i}^{n^{\prime}}(\nu)=$ $\sum_{j=1}^{\infty} t\left(s_{i}, s_{j}\right) \pi_{j}^{n}(\nu) \quad \forall \nu \in[0,1]$.

The intuition behind the definition is simple. Each realization $s_{i}$ under information structure $\boldsymbol{I}^{n^{\prime}}$ can be interpreted as being obtained from $\boldsymbol{I}^{n}$ by adding some noise through a process of randomization. In other words, the more informative structure is sufficient for the less informative.

Proposition B.2 $\boldsymbol{I}^{n}$ is more informative than $\boldsymbol{I}^{n^{\prime}} \Longleftrightarrow n>n^{\prime}$

Proof. We prove first that $n>n^{\prime} \Rightarrow \boldsymbol{I}^{n}$ is more informative than $\boldsymbol{I}^{n^{\prime}}$. For the sake of simplicity, consider the case where $n^{\prime}=n-1$. The argument can easily be extended to any $n^{\prime}<n$. The objective then is to find a transition probability matrix $t_{i, j}$ that replicates the probability distribution $\boldsymbol{\pi}^{n^{\prime}}(\nu)$ after being applied to $\boldsymbol{\pi}^{n}(\nu)$ for all $\nu$. It can be shown that only $n(n+1)$ coefficients need to be computed. The remaining ones trivially equal zero. The solution satisfies the following system of equations:

$$
\left[\begin{array}{cccc}
t_{11} & t_{12} & \ldots & t_{1 n+1} \\
t_{21} & t_{22} & \ldots & t_{2 n+1} \\
\ldots & \ldots & \ldots & \ldots \\
t_{n 1} & t_{n 2} & \ldots & t_{n n+1}
\end{array}\right]\left[\begin{array}{c}
\left(\begin{array}{c}
n \\
n
\end{array}\right) \nu^{n} \\
\left(\begin{array}{c}
n \\
n-1
\end{array}\right) \nu^{n-1}(1-\nu) \\
\vdots \\
\left(\begin{array}{l}
n \\
0
\end{array}\right)(1-\nu)^{n}
\end{array}\right]=\left[\begin{array}{c}
\left(\begin{array}{c}
n-1 \\
n-1
\end{array}\right) \nu^{n-1} \\
\left(\begin{array}{c}
n-1 \\
n-2
\end{array}\right) \nu^{n-2}(1-\nu) \\
\vdots \\
\left(\begin{array}{c}
n-1 \\
0
\end{array}\right)(1-\nu)^{n-1}
\end{array}\right]
$$

We let the reader check that the matrix

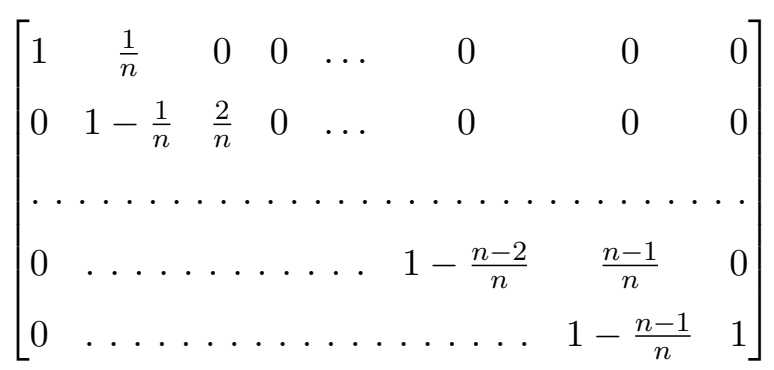

is a solution of system (B.1). 
It remains to be shown that if $\boldsymbol{I}^{n}$ is more informative than $\boldsymbol{I}^{n^{\prime}}$ then $n>n^{\prime}$. Assume that $n^{\prime}=n+1$. If the hypothesis is true, it must be the case then that there exists a solution for the following system of equations:

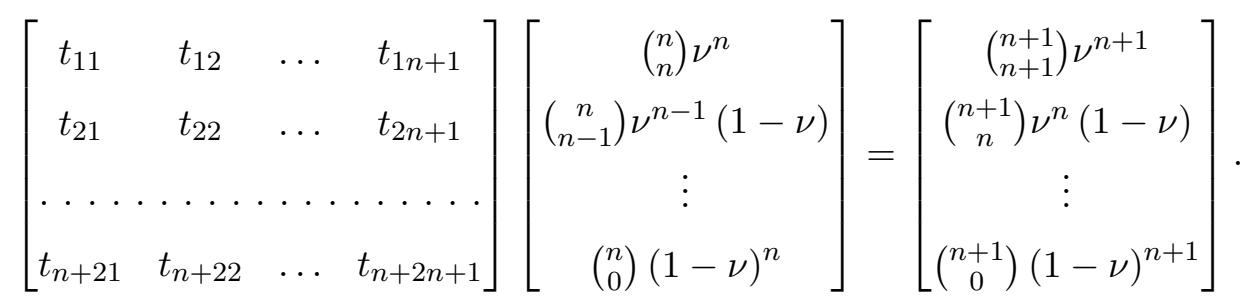

The first equation implies that a linear combination of $\nu^{0}, \nu, \ldots, \nu^{n-1}$ and $\nu^{n}$ must equal $\nu^{n+1}$ for all $\nu$ in the interval $[0,1]$. This is clearly not possible. The same argument holds for any $n^{\prime}>n+1$.

It is intuitive that a higher number of binary signals entails more precise information. We have showed above that this is true in a similar setting to the one described in the main text, and when the Blackwell's criterion is used to compare information structures. Unfortunately, the former criterion cannot be applied to our baseline model. In that framework, the degree of informativeness is endogenously determined. 


\section{Graphs}

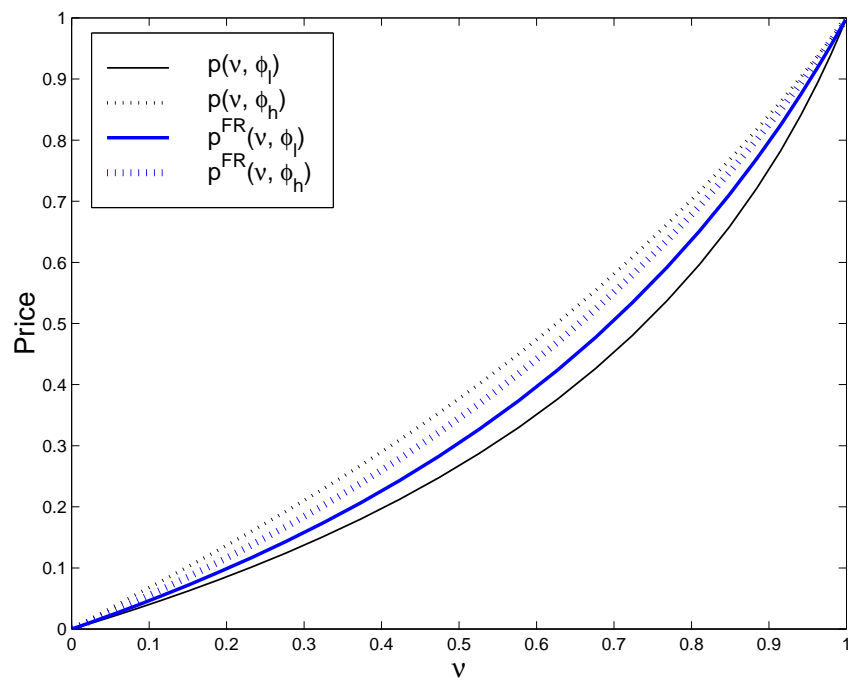

Figure 2: Equilibrium price under full and partial information 


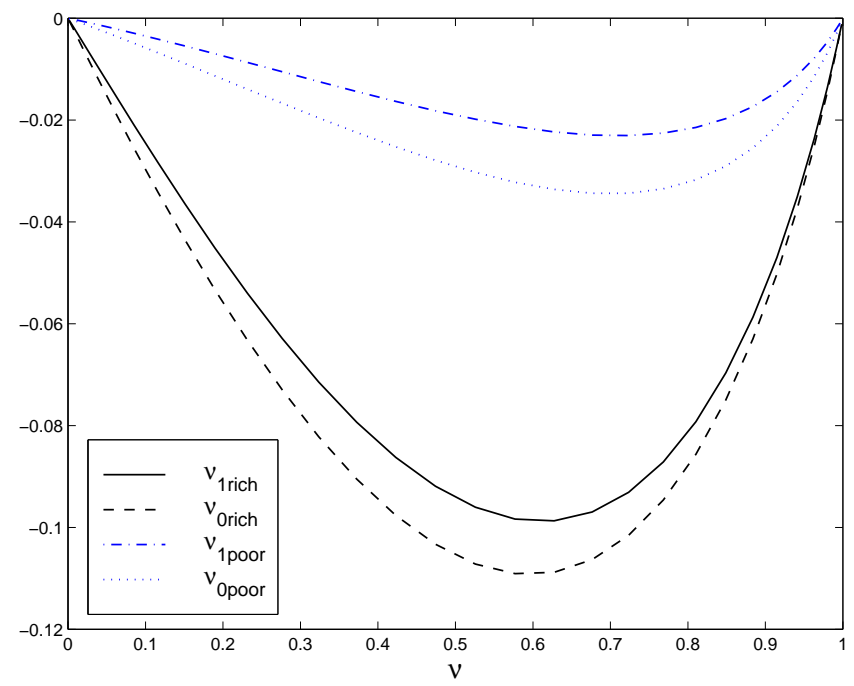

Figure 3: Difference between individual beliefs and actual realizations of $\nu$ for the case $\phi=\phi_{l}$

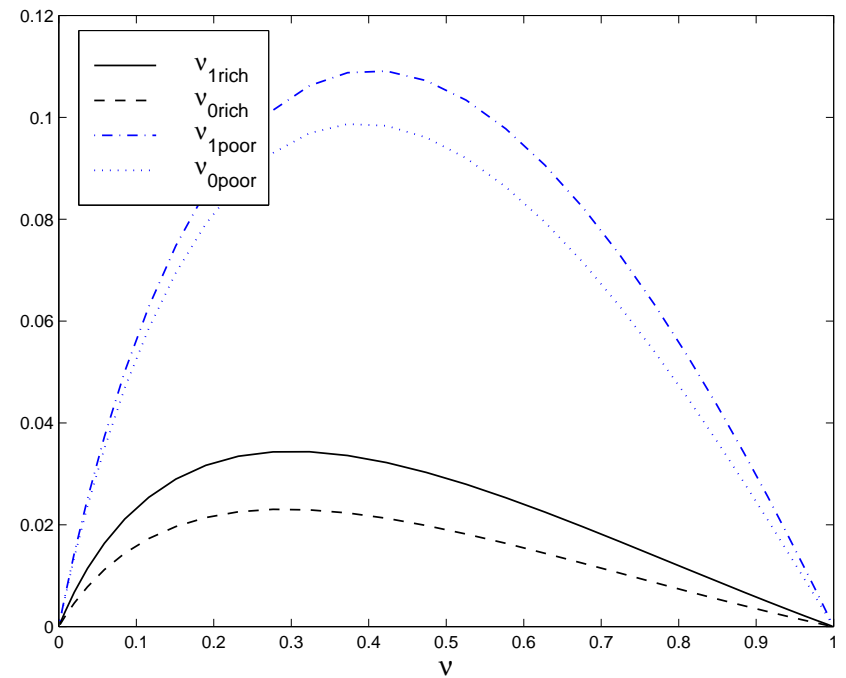

Figure 4: Difference between individual beliefs and actual realizations of $\nu$ for the case $\phi=\phi_{h}$ 


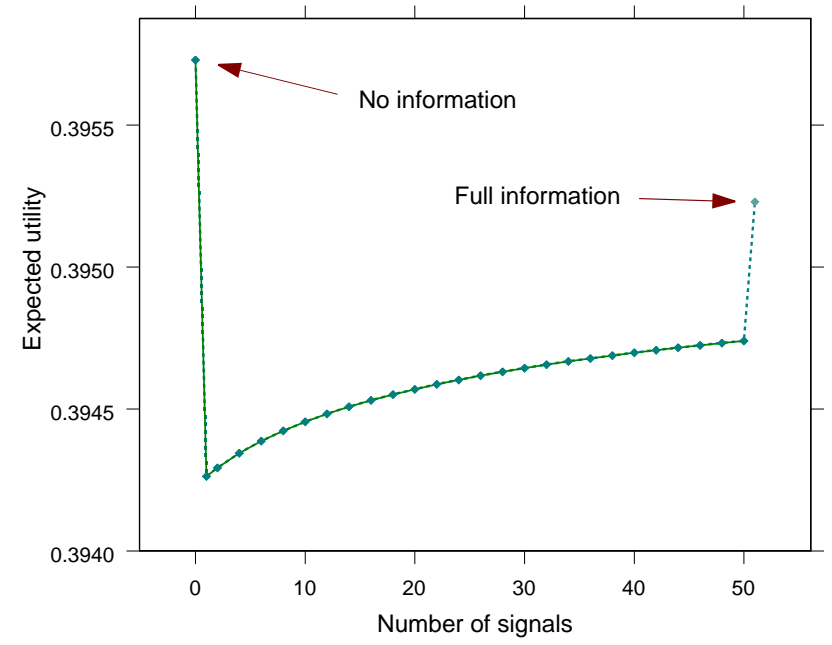

Figure 5: Welfare of rich agents as a function of the number of signals

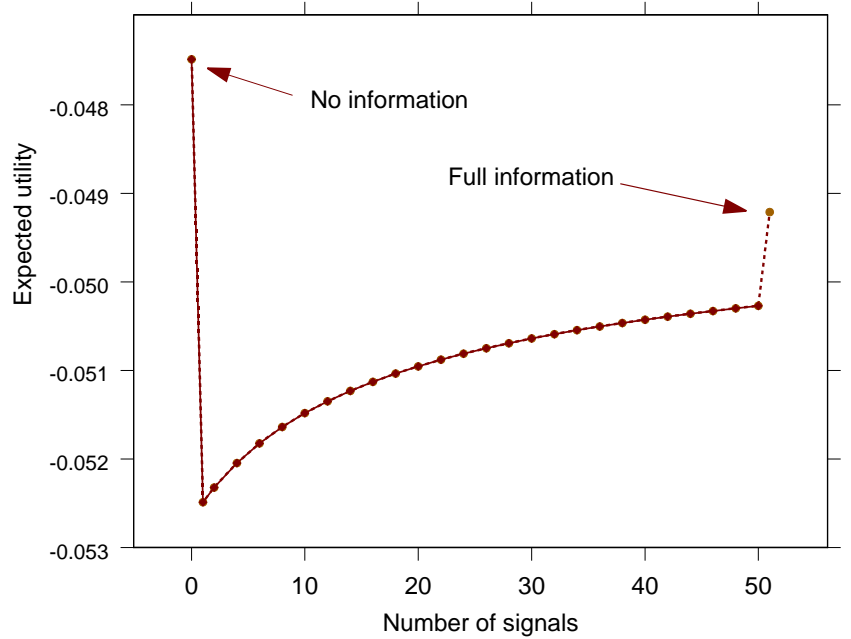

Figure 6: Welfare of poor agents as a function of the number of signals 


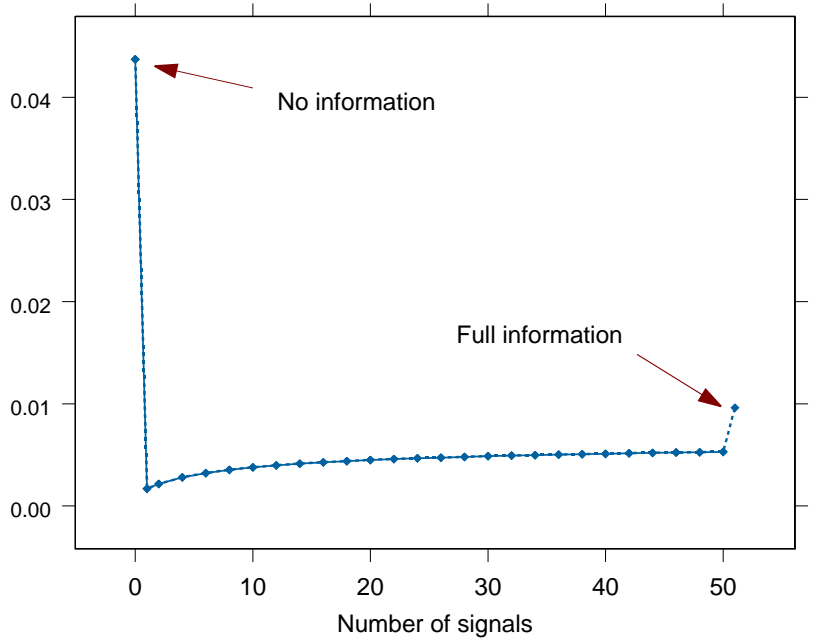

Figure 7: Aggregate volume of trade as a function of the number of signals

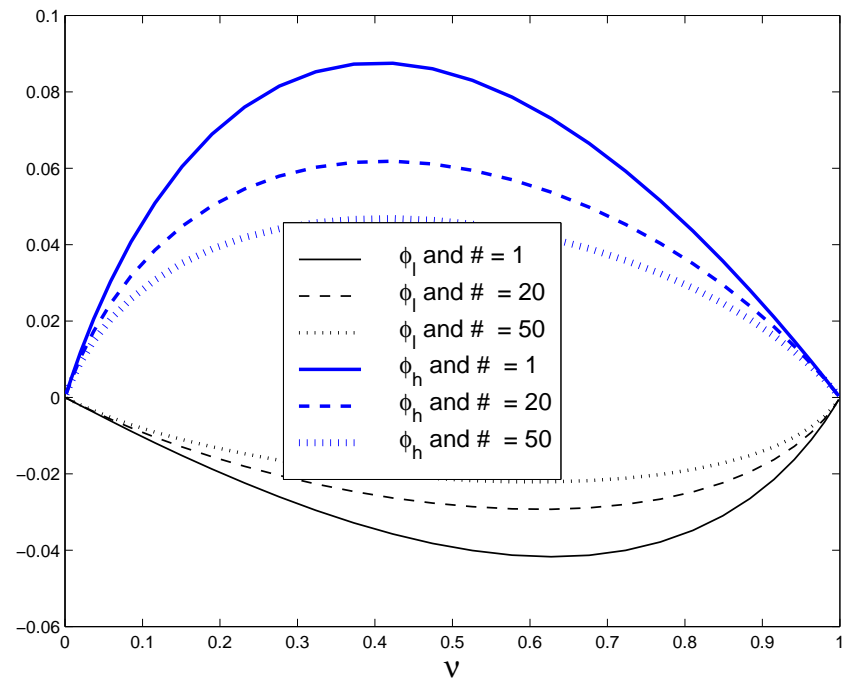

Figure 8: Difference between average beliefs and actual realizations of $\nu$ as a function of the number of signals 


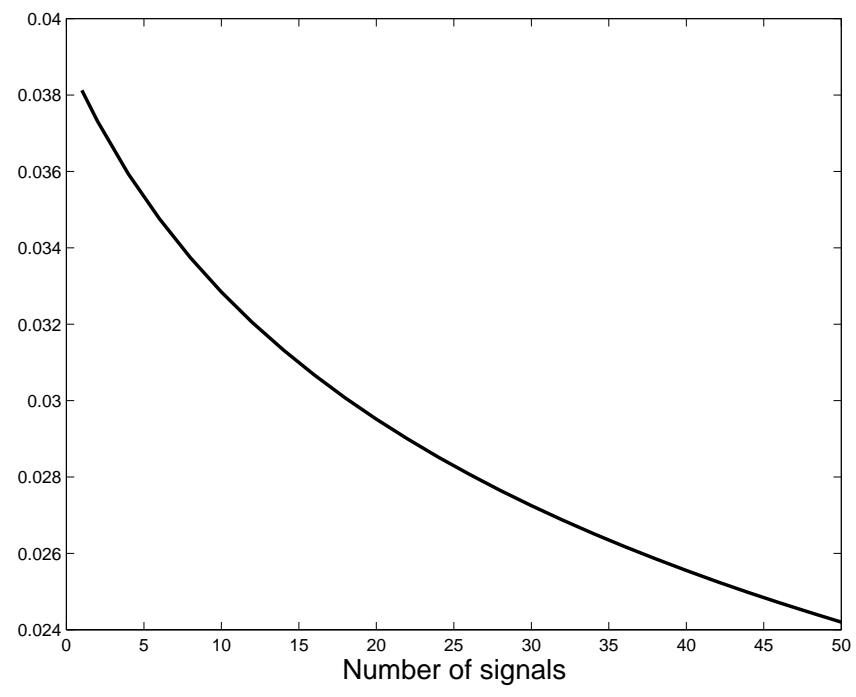

Figure 9: Precision of beliefs as a function of the number of signals 